\title{
Effect of fermented Rhus verniciflua stokes extract on liver function parameters in healthy Korean adults: a double-blind randomized controlled trial
}

\author{
Jung Hyun Kwak¹, Hyo-Jeong Lee², Seok-Tae Jeong ${ }^{3}$, Ju Yeon Lee ${ }^{1}$, Minho Lee ${ }^{4}$ and Jean Kyung Paik ${ }^{* *}$
}

\begin{abstract}
Background: Fermented Rhus verniciflua Stokes extract (FRVE) reported an anti-hepatic lipidemic property mediated by the upregulation of AMP-activated protein kinase (AMPK) in cell and animal models. However, it remains unclear whether there is an effect of FRVE on liver disease-related parameters and serum lipid levels in humans. We investigated the effects of FRVE intake for 12 weeks on liver disease-related parameters and serum lipid profiles in Korean adults.

Methods: A randomized, double-blind, placebo-controlled study was conducted among 79 subjects. An aqueous extract of fermented Rhus verniciflua Stokes that was filtered and fermented was prepared. For 12 weeks, the test group ( $n=39$ ) consumed two capsules of FRVE (main components: fustin $129 \mathrm{mg}$ and fisetin $59 \mathrm{mg}$ ) once daily. The control group $(n=40)$ consumed two placebo pills (main component: lactose $627.0 \mathrm{mg}$ ) once daily. A 1:1 randomization of control and test was performed using computer-generated randomization. Both before and after FRVE intake, anthropometric parameters, liver function-related parameters, and clinical laboratory parameters were measured. The effects between the test and control groups were compared using the Mann-Whitney $U$ test and independent $t$-test, and the difference between baseline and follow-up values was compared using Wilcoxon ranksum test and paired $t$-test.
\end{abstract}

Results: There was no significant difference when comparing the change values of liver disease-related parameters and serum lipid profiles in between groups.

Conclusions: In our study, we did not confirm the significance in liver function parameters and serum lipid profiles. Trial registration: The study protocol was registered in the Clinical Research Information Service (CRIS: https://cris. nih.go.kr/cris/index.jsp) under number KCT0005687. Registered on 2 December 2020

Keywords: Fermented Rhus verniciflua Stokes extract, Liver function, Randomized double-blind trial, Korean adult

\footnotetext{
* Correspondence: jkpaik@eulji.ac.kr

'Department of Food and Nutrition, Eulji University, Seongnam 13135, Gyeonggi-do, Republic of Korea

Full list of author information is available at the end of the article
}

(c) The Author(s). 2021 Open Access This article is licensed under a Creative Commons Attribution 4.0 International License, which permits use, sharing, adaptation, distribution and reproduction in any medium or format, as long as you give appropriate credit to the original author(s) and the source, provide a link to the Creative Commons licence, and indicate if changes were made. The images or other third party material in this article are included in the article's Creative Commons licence, unless indicated otherwise in a credit line to the material. If material is not included in the article's Creative Commons licence and your intended use is not permitted by statutory regulation or exceeds the permitted use, you will need to obtain permission directly from the copyright holder. To view a copy of this licence, visit http://creativecommons.org/licenses/by/4.0/. The Creative Commons Public Domain Dedication waiver (http://creativecommons.org/publicdomain/zero/1.0/) applies to the data made available in this article, unless otherwise stated in a credit line to the data. 


\section{Background}

Based on the Korea National Health and Nutrition Survey data from 1998 to 2017, a recent study reported that the prevalence of nonalcoholic fatty liver disease (NAFL D) is increasing rapidly, especially in men, estimating that NAFLD would reach $44 \%$ by 2035 [1]. Subjects with NAFLD have a higher mortality rate from liver diseases or other causes, and the risk of cardiovascular disease and chronic kidney disease is also increased compared to that in subjects without NAFLD [2, 3]. Therefore, prevention and treatment in subjects with NAFLD are important. But, since there is no registered drug for the treatment of NAFLD, diet and exercise are considered very important treatment methods [4].

Rhus verniciflua Stokes (RVS) is known by the common name lacquer tree, which is an Asian tree species of the Anacardiaceae family and cultivated in regions of China, Japan, and Korea [5]. The sap that comes out of a wound on the bark of a lacquer tree is called lacquer, and lacquer has been widely used for food and medicine since ancient times. The lacquer sap contains various active ingredients such as urushiol, fisetin, fustin, and butein, but urushiol is an allergic ingredient and causes connection dermatitis, so the range of use of lacquer was very limited [6]. However, in Korea, regulations on the use of urshiol-removed lacquer tree extract have been relaxed since 2004. So, various methods to remove urushiol have been developed and used [7].

Fermented Rhus verniciflua Stokes extract (FRVE) is a food that is free of urushiol allergens and contains two kinds of flavonoids such as fustin and fisetin. FRVE has been reported to have anti-hepatic lipidemic effects through the activation of AMPK [7]. Furthermore, Lee et al. reported in an animal model, in which the formation of a nonalcoholic fatty liver was induced by a highfat diet, that ingestion of FRVE reduced liver-related parameters, such as alanine aminotransferase (ALT) and aspartate aminotransferase (AST), and reduced lipidrelated parameters such as total cholesterol (TC) and triglycerides (TG) [8]. However, the effect of FRVE intake on liver function parameters has not been identified in humans. Therefore, we investigated the effect of intake of FRVE for 12 weeks on liver disease-related parameters and lipid profiles in Korean adults.

\section{Methods}

Preparation of fermented Rhus verniciflua Stokes extracts For the FRVE used in this study, $300 \mathrm{~kg}$ of lacquer bark (Okcheonsan, Chungbuk, Korea) was cut into $2 \times 2 \mathrm{~cm}$ pieces and placed in a bag that allows moisture to pass through. In order to absorb moisture in the bark of the sumac tree, it was immersed in water for 1 day and drained for 1 day. The lacquer bark was removed and transferred to a 5-L mushroom cultivation bag, and a filter was attached and sterilized at $121^{\circ} \mathrm{C}$ for $100 \mathrm{~min}$. Then, the FRVE was prepared by mixing and inoculating a long-lived mushroom starter (Fomitella fraxinea) prepared according to the method of Choi et al. [9], culturing at $21^{\circ} \mathrm{C}$ for 30 days, and drying it with hot air at $50^{\circ} \mathrm{C}$. Ten times distilled water was added to $100 \mathrm{~g}$ of fermented Rhus veniciflua Stokes (FRV) ground with a mixer. Its weight was measured, and it was put into an autoclave and extracted for $8 \mathrm{~h}$ at an interval of $1 \mathrm{~h}$ at 80-120 ${ }^{\circ} \mathrm{C}$. Thereafter, water was added to correct the amount of evaporated moisture and adjusted to the same weight. The crude extract was centrifuged $\left(4{ }^{\circ} \mathrm{C}\right.$, $12,000 \times \mathrm{g}, 30 \mathrm{~min}$ ) and filtered (Filter paper No. 2, What$\operatorname{man}^{\mathrm{Tm}}$, GE Healthcare Co., Buckinghamshire, UK). The solid content was obtained by drying $200 \mathrm{~mL}$ of the filtered FRVE at $105{ }^{\circ} \mathrm{C}$ and was calculated as the solid content for $100 \mathrm{~g}$ of FRVE. We used the FRVE samples that went through the above standardization process provided by the Rural Development Administration.

\section{Study subjects}

Subjects were recruited from June to October 2020. Subjects were recruited from local advertisements in the Eulji University Seongnam Campus area. Clinical trial recruitment posters contained brief information about the trial and were posted in Eulji University Seongnam Campus area after institutional review board approval. Subjects included were (1) male or female subjects with age $\geq 20$ years, (2) subjects without any diseases, and (3) subjects who agreed to participate in the clinical trial and signed the informed consent form. Subjects were excluded if they had any diagnosis of renal disease, liver disease, cardiovascular disease, and cancer or consumed any disease-related drugs.

This study was conducted as a double-blind randomized controlled design. We explained this study to the subjects and obtained written consent before initiating the study. The test and placebo capsules were provided and blinded by the Rural Development Administration (Jeollabuk-do, Republic of Korea). The 500-mg test capsule is composed of FRVE $400 \mathrm{mg}$, crystalline cellulose $75 \mathrm{mg}$, calcium carboxymethyl cellulose $15 \mathrm{mg}$, silica 5 $\mathrm{mg}$, and magnesium stearate $5 \mathrm{mg}$. The $500-\mathrm{mg}$ placebo capsule is composed of lactose powder $313.5 \mathrm{mg}$, caramel color $75 \mathrm{mg}$, gardenia blue color $1.5 \mathrm{mg}$, crystalline cellulose $105 \mathrm{mg}$, and magnesium stearate $5 \mathrm{mg}$. The randomization sequence was generated by the Rural Development Administration using a computer-generated randomization (control:test $=1: 1$ ) (https://www. randomizer.org/). To maintain the blinding, the appearance of the test and placebo product was identical. For 12 weeks, the test group $(n=39)$ consumed two capsules of FRVE (main components: fustin $129 \mathrm{mg}$ and fisetin $59 \mathrm{mg})$ once per day. The control group $(n=40)$ 
consumed two placebo pills (main components: lactose $627.0 \mathrm{mg}$ ) once per day. Subjects visited the Clinical $\mathrm{Nu}$ trition Laboratory located in Eulji University 3 times during the study period. Scheduled assessments were conducted at screening and baseline (week 0 ) and at the end of weeks 6 and 12. Participants were encouraged to maintain their usual lifestyle and dietary habits and to keep a food diary. Compliance was assessed by counting the remaining packs at 12 weeks, and if more than $80 \%$ of the packs were consumed, compliance was considered acceptable.

\section{Human dose calculation}

In the experimental study, the conditions for using a dose of $2000 \mathrm{mg} / \mathrm{kg}$ or less of FRVE were established based on the results of the toxicity test conducted by the ChemOn Preclinical Research Center (Gyeonggi-do,

Table 1 Comparison of the clinical characteristics of the subjects at baseline

\begin{tabular}{|c|c|c|c|}
\hline \multirow[t]{2}{*}{ Characteristic } & \multicolumn{3}{|l|}{ Group (total = 52) } \\
\hline & Control $(n=25)$ & FRVE $(n=27)$ & $p$ \\
\hline Sex, no. (\%) of participant & & & 0.893 \\
\hline Men & $5(20.0)$ & $5(18.5)$ & \\
\hline Women & $20(80.0)$ & $22(81.5)$ & \\
\hline Age (years) & $33.24( \pm 3.06)$ & $33.07( \pm 2.92)$ & 0.969 \\
\hline Height (cm) & $162.55( \pm 1.58)$ & $163.37( \pm 1.19)$ & 0.679 \\
\hline Weight (kg) & $59.36( \pm 2.66)$ & $63.69( \pm 2.06)$ & 0.201 \\
\hline Waist (cm) & $74.61( \pm 2.36)$ & $79.87( \pm 2.35)$ & 0.121 \\
\hline Hip (cm) & $94.66( \pm 1.62)$ & $95.67( \pm 1.46)$ & 0.641 \\
\hline $\mathrm{SBP}(\mathrm{mmHg})$ & $111.48( \pm 2.82)$ & $111.93( \pm 2.45)$ & 0.905 \\
\hline $\mathrm{DBP}(\mathrm{mmHg})$ & $68.40( \pm 2.20)$ & $69.96( \pm 2.08)$ & 0.608 \\
\hline \multicolumn{4}{|l|}{ Blood parameters } \\
\hline Glucose (mg/dL) & $92.64( \pm 2.32)$ & $89.78( \pm 1.67)$ & 0.337 \\
\hline Insulin (uU/mL) & $10.99( \pm 2.89)$ & $16.44( \pm 3.32)$ & 0.074 \\
\hline Albumin (g/dL) & $4.94( \pm 0.06)$ & $4.94( \pm 0.04)$ & 0.964 \\
\hline WBC $\left(\mathrm{mm}^{3}\right)$ & $6.31( \pm 0.30)$ & $6.37( \pm 0.25)$ & 0.885 \\
\hline $\mathrm{RBC}\left(\mathrm{mm}^{3}\right)$ & $4.60( \pm 0.09)$ & $4.46( \pm 0.07)$ & 0.205 \\
\hline $\mathrm{Hb}(\mathrm{g} / \mathrm{dL})$ & $13.82( \pm 0.25)$ & $13.67( \pm 0.22)$ & 0.657 \\
\hline $\mathrm{HCT}(\%)$ & $41.09( \pm 0.62)$ & $40.59( \pm 0.57)$ & 0.555 \\
\hline $\operatorname{PLT}\left(10^{3} / \mathrm{uL}\right)$ & $258.36( \pm 11.88)$ & $235.67( \pm 11.64)$ & 0.179 \\
\hline AST (IU/L) & $20.48( \pm 1.44)$ & $20.15( \pm 1.12)$ & 0.995 \\
\hline ALT (IU/L) & $17.67( \pm 2.71)$ & $16.63( \pm 1.62)$ & 0.949 \\
\hline AST/ALT ratio & $1.44( \pm 0.10)$ & $1.40( \pm 0.09)$ & 0.765 \\
\hline GGT (mg/dL) & $18.32( \pm 2.02)$ & $21.59( \pm 3.06)$ & 0.384 \\
\hline ALP (IU/L) & $64.56( \pm 2.92)$ & $66.00( \pm 4.21)$ & 0.783 \\
\hline $\mathrm{TC}(\mathrm{mg} / \mathrm{dL})$ & $190.84( \pm 5.08)$ & $179.44( \pm 5.69)$ & 0.144 \\
\hline $\mathrm{TG}(\mathrm{mg} / \mathrm{dL})$ & $125.76( \pm 23.55)$ & $138.74( \pm 22.23)$ & 0.589 \\
\hline $\mathrm{HDL}-\mathrm{C}(\mathrm{mg} / \mathrm{dL})$ & $60.44( \pm 2.93)$ & $62.59( \pm 3.48)$ & 0.641 \\
\hline LDL-C (mg/dL) & $105.25( \pm 5.39)$ & $92.26( \pm 5.61)$ & 0.102 \\
\hline MDA (pmol/mL) & $321.30( \pm 70.57)$ & $225.69( \pm 40.30)$ & 0.373 \\
\hline Adiponectin (ug/uL) & $6.89( \pm 0.53)$ & $6.57( \pm 0.40)$ & 0.628 \\
\hline Oxi-LDL (U/L) & $40,718.78( \pm 3307.54)$ & $35,885.09( \pm 2434.90)$ & 0.240 \\
\hline
\end{tabular}

Independent $t$-test was used for continuous variables (mean $\pm \mathrm{SE}$ ) and chi-square test was used for categorical variables $(n, \%)$

AST aspartate aminotransferase, ALT alanine aminotransferase, ALP alkaline phosphatase, DBP diastolic blood pressure, GGT $\gamma$-glutamyl transferase, $H C T$ hematocrit, $H b$ hemoglobin, $H D L-C$ high-density lipoprotein cholesterol, LDL-C low-density lipoprotein cholesterol, MDA malondialdehyde, PLT platelet, RBC red blood cells, SBP systolic blood pressure, TC total cholesterol, TG triglycerides, WBC white blood cells 
Korea), and the effective concentration of FRVE in this study was derived based on a previous study $[8,10,11]$.

\section{Sample size}

To calculate the number of clinical trial subjects in this study, the average value of 32.7 for those with $25 \mathrm{IU} / \mathrm{L}$ or more among the general AST data presented by Kim et al.'s study was referenced [12]. Since a $10 \%$ reduction in this average value can be regarded as an effective reduction value, the average difference between the treatment group and the control group was assumed to be 3.27, and the standard deviation was assumed to be 4.0 . Assuming a significance level of $5 \%$ and power of $80 \%$, $Z_{1 \alpha / 2}=1.96$ (two-tailed test), $Z_{1-\beta}=0.845, D_{\mathrm{t}}-D_{\mathrm{c}}=3.27$, and $\sigma=4.0$ were substituted into the formula. The number of subjects was calculated considering the dropout rate of $20 \%$ and the compliance rate of $80 \%$. The number of subjects in each group was 35 or more, and a total of 79 subjects were recruited.

\section{Anthropometric parameters and blood collection}

Body weight and height were measured in the morning in unclothed subjects without shoes to calculate the body mass index (BMI; $\mathrm{kg} / \mathrm{m}^{2}$ ). Blood pressure (BP) was measured at baseline and at the 12-week follow-up visits using a sphygmomanometer (Omron HEM-7120, OMRON Co., Ltd, Japan). We obtained three BP measurements at each visit, and differences among the three systolic blood pressure (SBP) readings were always < 5 $\mathrm{mmHg}$. Participants were instructed not to smoke or drink alcohol for at least $30 \mathrm{~min}$ before measuring BP. Patients were also instructed to undergo a 12-h fasting period before the initial blood draw and before the follow-up visits. Venous blood specimens were collected in EDTA-treated plain tubes and then centrifuged to produce plasma or serum, which was subsequently stored at $-70{ }^{\circ} \mathrm{C}$ until analysis.

\section{Liver function-related parameters and lipid profile}

The serum levels of AST, ALT, and gamma-glutamyl transferase (GGT) were measured using aspartate aminotransferase, alanine aminotransferase, and y-glutamyltransferase kits (Roche, Germany) and a Cobas 800 analyzer (Roche, Germany), according to the manufacturer's instructions. Alkaline phosphatase levels were measured using an ALP2 kit (Roche, Germany) and a

Table 2 Liver function-related parameters and serum lipid levels before and 12 weeks after consuming FRVE

\begin{tabular}{|c|c|c|c|c|c|c|c|}
\hline & \multicolumn{7}{|c|}{ Group (total $=52$ ) } \\
\hline & \multicolumn{3}{|c|}{ Control $(n=25)$} & \multicolumn{3}{|l|}{ FRVE $(n=27)$} & \multirow[b]{2}{*}{$p^{\mathrm{b}}$} \\
\hline & 0 weeks & 12 weeks & $p^{a}$ & 0 weeks & 12 weeks & $p^{a}$ & \\
\hline AST (IU/L)* & $20.48( \pm 1.44)$ & $19.88( \pm 1.24)$ & 0.778 & $20.15( \pm 1.12)$ & $20.85( \pm 1.75)$ & 0.836 & \\
\hline$\Delta$ change & $-0.60( \pm 1.38)$ & & & $0.70( \pm 0.96)$ & & & 0.436 \\
\hline ALT (IU/L)* & $17.67( \pm 2.71)$ & $17.25( \pm 1.96)$ & 0.500 & $16.63( \pm 1.62)$ & $16.89( \pm 1.90)$ & 0.928 & \\
\hline$\Delta$ change & $-0.42( \pm 1.88)$ & & & $0.26( \pm 1.31)$ & & & 0.765 \\
\hline AST/ALT ratio & $1.44( \pm 0.10)$ & $1.36( \pm 0.10)$ & 0.095 & $1.40( \pm 0.09)$ & $1.38( \pm 0.08)$ & 0.832 & \\
\hline$\Delta$ change & $-0.09( \pm 0.05)$ & & & $-0.02( \pm 0.09)$ & & & 0.523 \\
\hline GGT (mg/dL)* & $18.32( \pm 2.02)$ & $17.48( \pm 1.99)$ & 0.241 & $21.59( \pm 3.06)$ & $19.44( \pm 2.88)$ & 0.063 & \\
\hline$\Delta$ change & $-0.84( \pm 0.70)$ & & & $-2.15( \pm 1.11)$ & & & 0.331 \\
\hline ALP (IU/L) & $64.56( \pm 2.92)$ & $63.08( \pm 2.50)$ & 0.289 & $66.00( \pm 4.21)$ & $65.31( \pm 4.28)$ & 0.608 & \\
\hline$\Delta$ change & $-1.48( \pm 1.36)$ & & & $-0.63( \pm 1.21)$ & & & 0.642 \\
\hline $\mathrm{TC}(\mathrm{mg} / \mathrm{dL})$ & $190.84( \pm 5.07)$ & $187.20( \pm 4.93)$ & 0.231 & $179.44( \pm 5.69)$ & $176.56( \pm 5.89)$ & 0.377 & \\
\hline$\Delta$ change & $-3.64( \pm 2.96)$ & & & $-2.89( \pm 3.22)$ & & & 0.865 \\
\hline TG $(\mathrm{mg} / \mathrm{dL})^{*}$ & $125.76( \pm 23.55)$ & $97.68( \pm 11.12)$ & 0.073 & $138.74( \pm 22.23)$ & $110.70( \pm 17.26)$ & 0.046 & \\
\hline$\Delta$ change & $-28.08( \pm 13.64)$ & & & $-28.04( \pm 14.20)$ & & & 0.998 \\
\hline HDL-C (mg/dL) & $60.44( \pm 2.93)$ & $62.32( \pm 2.44)$ & 0.184 & $62.59( \pm 3.48)$ & $62.52( \pm 3.23)$ & 0.957 & \\
\hline$\Delta$ change & $1.88( \pm 1.38)$ & & & $-0.07( \pm 1.37)$ & & & 0.320 \\
\hline LDL-C (mg/dL) & $105.25( \pm 5.39)$ & $105.34( \pm 5.01)$ & 0.976 & $92.26( \pm 5.61)$ & $95.73( \pm 5.71)$ & 0.306 & \\
\hline$\Delta$ change & $0.10( \pm 3.21)$ & & & $3.47( \pm 3.32)$ & & & 0.469 \\
\hline
\end{tabular}


Cobas 800 analyzer. Fasting serum TC, high-density lipoprotein (HDL) cholesterol, and TG levels were measured using commercially available kits and a Cobas 8000 analyzer. Low-density lipoprotein (LDL) cholesterol was indirectly estimated in participants with serum triglyceride levels $<400 \mathrm{mg} / \mathrm{dL}$ using the Friedewald formula: LDL cholesterol $=\mathrm{TC}-(\mathrm{HDL}$ cholesterol $+(\mathrm{TG} / 5))$.

\section{Laboratory blood parameters}

Complete blood count (CBC), white blood cell (WBC) count $\left(10^{3} / \mu \mathrm{L}\right)$, red blood cell $(\mathrm{RBC})$ count $\left(10^{6} / \mu \mathrm{L}\right)$, platelet (PLT) count $\left(10^{3} / \mu \mathrm{L}\right)$, hematocrit (Hct) (\%), and hemoglobin $(\mathrm{Hb})$ level $(\mathrm{g} / \mathrm{dL})$ were measured using commercially available kits and the Sysmex analyzer (Sysmex, Japan). The levels of glucose, insulin, free fatty acid, blood urea nitrogen, and creatinine were measured using commercially available kits and the Cobas 8000 analyzer.

\section{Assessment of food intake}

The diet information was obtained using a 24-h recall method. All subjects received written and oral instructions by a dietitian on how to complete the 3-day ( 2 weekdays and 1 weekend day) dietary records. On the recording sheet, subjects wrote the kinds and amounts of food they consumed. Dietary energy values and nutrient content from all complete 3-day dietary records were calculated using the Computer-Aided Nutritional Analysis Program (CAN-Pro 5.0, Korean Nutrition Society, Seoul, Korea).

\section{Data analysis}

Statistical analysis was performed using Statistical Package for the Social Sciences (SPSS), version 20.0 for Windows (SPSS, Chicago, IL, USA). Skewed variables were logarithmically transformed for the statistical analyses. Categorized variables were tested using the chi-square test. Mann-Whitney $U$ test (nonparametric test) and independent $t$-test were used for comparing the effects between the test and control groups. Wilcoxon rank-sum test (nonparametric test) and paired $t$-test were used for comparing the baseline and follow-up values. In groups with $<20$ subjects, a nonparametric test was used. For descriptive purposes, mean values are presented using

Table 3 Liver function-related parameters and serum lipids before and 12 weeks after consuming FRVE among men

\begin{tabular}{|c|c|c|c|c|c|c|c|}
\hline & \multicolumn{7}{|c|}{ Group (total = 10) } \\
\hline & \multicolumn{3}{|l|}{ Control $(n=5)$} & \multicolumn{3}{|l|}{ FRVE $(n=5)$} & \multirow[b]{2}{*}{$p^{\mathbf{b}}$} \\
\hline & 0 weeks & 12 weeks & $p^{a}$ & 0 weeks & 12 weeks & $p^{a}$ & \\
\hline AST (IU/L)* & $22.40( \pm 2.98)$ & $24.60( \pm 4.40)$ & 0.273 & $25.80( \pm 3.14)$ & $30.00( \pm 7.33)$ & 0.465 & \\
\hline$\Delta$ change & $2.20( \pm 2.44)$ & & & $4.20( \pm 4.35)$ & & & 0.834 \\
\hline ALT (IU/L)* & $25.20( \pm 6.93)$ & $26.20( \pm 6.03)$ & 0.581 & $29.00( \pm 2.66)$ & $28.80( \pm 6.11)$ & 0.684 & \\
\hline$\Delta$ change & $1.00( \pm 1.70)$ & & & $-0.20( \pm 4.75)$ & & & 0.461 \\
\hline AST/ALT ratio & $1.03( \pm 0.14)$ & $0.99( \pm 0.11)$ & 0.500 & $0.90( \pm 0.09)$ & $1.02( \pm 0.04)$ & 0.225 & \\
\hline$\Delta$ change & $-0.04( \pm 0.06)$ & & & $0.12( \pm 0.11)$ & & & 0.175 \\
\hline GGT $(\mathrm{mg} / \mathrm{dL})^{*}$ & $20.40( \pm 3.04)$ & $20.60( \pm 2.16)$ & 0.891 & $35.00( \pm 10.89)$ & $27.60( \pm 7.81)$ & 0.068 & \\
\hline$\Delta$ change & $0.20( \pm 2.58)$ & & & $-7.40( \pm 3.79)$ & & & 0.169 \\
\hline ALP & $56.60( \pm 3.57)$ & $56.00( \pm 2.59)$ & 0.892 & $64.00( \pm 4.16)$ & $62.40( \pm 3.44)$ & 0.461 & \\
\hline$\Delta$ change & $-0.60( \pm 1.69)$ & & & $-1.60( \pm 3.53)$ & & & 0.916 \\
\hline $\mathrm{TC}(\mathrm{mg} / \mathrm{dL})$ & $194.20( \pm 12.87)$ & $191.60( \pm 12.50)$ & 0.498 & $176.80( \pm 11.32)$ & $169.40( \pm 15.50)$ & 0.225 & \\
\hline$\Delta$ change & $-2.60( \pm 10.67)$ & & & $-7.40( \pm 6.61)$ & & & 0.832 \\
\hline TG $(\mathrm{mg} / \mathrm{dL})^{*}$ & $152.20( \pm 72.55)$ & $113.40( \pm 24.49)$ & 0.893 & $275.40( \pm 58.19)$ & $144.40( \pm 37.27)$ & 0.043 & \\
\hline$\Delta$ change & $-38.8( \pm 50.50)$ & & & $-131.0( \pm 43.32)$ & & & 0.076 \\
\hline HDL-C (mg/dL) & $51.00( \pm 3.82)$ & $56.20( \pm 4.75)$ & 0.223 & $43.60( \pm 5.56)$ & $47.60( \pm 3.47)$ & 0.138 & \\
\hline$\Delta$ change & $5.20( \pm 3.18)$ & & & $4.00( \pm 2.35)$ & & & 0.916 \\
\hline LDL-C (mg/dL) & $112.76( \pm 20.55)$ & $112.72( \pm 12.61)$ & 0.893 & $78.12( \pm 9.75)$ & $92.92( \pm 13.76)$ & 0.080 & \\
\hline$\Delta$ change & $-0.04( \pm 10.47)$ & & & $14.80( \pm 7.19)$ & & & 0.175 \\
\hline
\end{tabular}

Data are presented as mean $\pm \mathrm{SE}$. Data are presented as mean $\pm \mathrm{SE}$

AST aspartate aminotransferase, $A L T$ alanine aminotransferase, ALP alkaline phosphatase, GGT $\gamma$-glutamyl transferase, $H D L-C$ high-density lipoprotein cholesterol,

$L D L-C$ low-density lipoprotein cholesterol, $T C$ total cholesterol, $T G$ triglycerides

*Tested using logarithmic transformation

${ }^{a} p$-values were calculated using the Wilcoxon test comparing the baseline values ( 0 weeks)

${ }^{\mathrm{b}} p$-values were calculated using the Mann-Whitney test when comparing the "change" values 
untransformed values. Results are expressed as mean \pm standard error (SE). A two-tailed $p$-value $<0.05$ was considered statistically significant. We used AST as the primary efficacy outcome, and other liver indicators such as ALT and GGT, and lipid parameters were used as secondary efficacy outcomes.

\section{Results}

\section{Characteristics of study participants}

Altogether, among the enrolled subjects $(n=79$, test $=$ 39 , and control $=40), 27$ dropped out due to refusal to revisit amidst the coronavirus disease pandemic $(n=$ 10 ), personal reasons including employment commitments and relocating $(n=5)$, and suboptimal compliance with FRVE intake $(n=12)$. Finally, 52 subjects (test $=27$, control $=25)$ completed the study $($ CONSORT 2010 Flow Diagram).

Table 1 shows the clinical characteristics of participants in the control and the test group at baseline. There were no significant differences between the two groups in the distribution of age, sex, height, weight, SBP, diastolic blood pressure (DBP), and blood parameters.

\section{Effect of FRVE supplementation on liver function and lipid metabolism parameters}

Table 2 shows the effect of FRVE supplementation on liver function and lipid metabolism parameters. The net changes in liver function and lipid metabolism parameters were not significantly different between the two groups.

When analyzing liver function and lipid metabolism parameters by sex, no significant differences were found in the liver function and lipid metabolism parameters (Tables 3 and 4).

\section{Effect of FRVE supplementation on laboratory blood parameters}

Table 5 shows the effect of FRVE supplementation on laboratory blood parameters. PLT significantly increased in the test group, and the changes in PLT were significantly different between the two groups.

\section{Effect of FRVE supplementation on dietary parameters}

Table 6 shows the effect of FRVE supplementation on dietary parameters. The net changes in dietary

Table 4 Liver function-related parameters and serum lipids before and 12 weeks after consuming FRVE among women

\begin{tabular}{|c|c|c|c|c|c|c|c|}
\hline & \multicolumn{7}{|c|}{ Group (total = 42) } \\
\hline & \multicolumn{3}{|l|}{ Control $(n=20)$} & \multicolumn{3}{|l|}{ FRVE $(n=22)$} & \multirow[b]{2}{*}{$p^{\mathbf{b}}$} \\
\hline & 0 weeks & 12 weeks & $p^{a}$ & 0 weeks & 12 weeks & $p^{a}$ & \\
\hline AST (IU/L)* & $20.00( \pm 1.66)$ & $18.70( \pm 1.02)$ & 0.581 & $18.86( \pm 1.03)$ & $18.77( \pm 1.10)$ & 0.776 & \\
\hline$\Delta$ change & $-1.30( \pm 1.60)$ & & & $-0.09( \pm 0.64)$ & & & 0.699 \\
\hline ALT (IU/L)* & $15.68( \pm 2.83)$ & $14.89( \pm 1.61)$ & 0.669 & $13.82( \pm 1.29)$ & $14.18( \pm 1.41)$ & 0.798 & \\
\hline$\Delta$ change & $-0.79( \pm 2.34)$ & & & $0.36( \pm 1.28)$ & & & 0.818 \\
\hline AST/ALT ratio & $1.55( \pm 0.11)$ & $1.45( \pm 0.12)$ & 0.119 & $1.52( \pm 0.10)$ & $1.46( \pm 0.09)$ & 0.646 & \\
\hline$\Delta$ change & $-0.10( \pm 0.06)$ & & & $-0.05( \pm 0.11)$ & & & 0.235 \\
\hline GGT $(\mathrm{mg} / \mathrm{dL})^{*}$ & $17.80( \pm 2.42)$ & $16.70( \pm 2.42)$ & 0.023 & $18.55( \pm 2.59)$ & $17.59( \pm 3.01)$ & 0.112 & \\
\hline$\Delta$ change & $-1.10( \pm 0.64)$ & & & $-0.95( \pm 0.93)$ & & & 0.136 \\
\hline ALP & $66.55( \pm 3.42)$ & $64.85( \pm 2.95)$ & 0.320 & $66.45( \pm 5.11)$ & $66.05( \pm 5.21)$ & 0.755 & \\
\hline$\Delta$ change & $-1.70( \pm 1.67)$ & & & $-0.41( \pm 1.30)$ & & & 0.805 \\
\hline $\mathrm{TC}(\mathrm{mg} / \mathrm{dL})$ & $190.00( \pm 5.64)$ & $186.10( \pm 5.46)$ & 0.180 & $180.05( \pm 6.60)$ & $178.18( \pm 6.45)$ & 0.618 & \\
\hline$\Delta$ change & $-3.90( \pm 2.80)$ & & & $-1.86( \pm 3.68)$ & & & 0.667 \\
\hline TG $(\mathrm{mg} / \mathrm{dL})^{*}$ & $119.15( \pm 24.23)$ & $93.75( \pm 12.64)$ & 0.054 & $107.68( \pm 18.88)$ & $103.05( \pm 19.43)$ & 0.421 & \\
\hline$\Delta$ change & $-25.40( \pm 12.56)$ & & & $-4.64( \pm 9.17)$ & & & 0.203 \\
\hline HDL-C (mg/dL) & $62.80( \pm 3.36)$ & $63.85( \pm 2.76)$ & 0.494 & $66.91( \pm 3.51)$ & $65.91( \pm 3.52)$ & 0.525 & \\
\hline$\Delta$ change & $1.05( \pm 1.51)$ & & & $-1.00( \pm 1.55)$ & & & 0.769 \\
\hline LDL-C (mg/dL) & $103.37( \pm 4.77)$ & $103.50( \pm 5.50)$ & 0.968 & $95.63( \pm 6.42)$ & $96.40( \pm 6.43)$ & 0.831 & \\
\hline$\Delta$ change & $0.13( \pm 3.24)$ & & & $0.77( \pm 3.56)$ & & & 0.276 \\
\hline
\end{tabular}

Data are presented as mean $\pm \mathrm{SE}$

AST aspartate aminotransferase, $A L T$ alanine aminotransferase, ALP alkaline phosphatase, GGT $\gamma$-glutamyl transferase, $H D L-C$ high-density lipoprotein cholesterol,

$L D L-C$ low-density lipoprotein cholesterol, TC total cholesterol, TG triglycerides

*Tested using logarithmic transformation

a $p$-values were calculated using the Wilcoxon test comparing the baseline values ( 0 weeks)

b $p$-values were calculated using the Mann-Whitney test when comparing the "change" values 
Table 5 Laboratory blood parameters before and 12 weeks after consuming FRVE

\begin{tabular}{|c|c|c|c|c|c|c|c|}
\hline & \multicolumn{7}{|c|}{ Group (total = 52) } \\
\hline & \multicolumn{3}{|l|}{ Control $(n=25)$} & \multicolumn{3}{|l|}{ FRVE $(n=27)$} & \multirow[b]{2}{*}{$p^{\mathrm{b}}$} \\
\hline & 0 weeks & 12 weeks & $p^{\mathrm{a}}$ & 0 weeks & 12 weeks & $p^{a}$ & \\
\hline Glucose (mg/dL)* & $92.64( \pm 2.32)$ & $97.04( \pm 7.46)$ & 0.712 & $89.78( \pm 1.67)$ & $87.41( \pm 2.32)$ & 0.312 & \\
\hline$\Delta$ change & $4.40( \pm 6.37)$ & & & $-2.37( \pm 2.68)$ & & & 0.319 \\
\hline Insulin $(\mu \mathrm{U} / \mathrm{mL})^{*}$ & $10.99( \pm 2.89)$ & $9.78( \pm 1.61)$ & 0.822 & $16.44( \pm 3.32)$ & $10.62( \pm 1.72)$ & 0.046 & \\
\hline$\Delta$ change & $-1.22( \pm 2.03)$ & & & $-5.81( \pm 3.25)$ & & & 0.245 \\
\hline HOMA-IR & $2.52( \pm 0.64)$ & $2.49( \pm 0.49)$ & 0.948 & $3.62( \pm 0.70)$ & $2.22( \pm 0.32)$ & 0.043 & \\
\hline$\Delta$ change & $-0.03( \pm 0.51)$ & & & $-1.41( \pm 0.66)$ & & & 0.112 \\
\hline FFA & $604.20( \pm 74.01)$ & $547.96( \pm 57.26)$ & 0.440 & $581.19( \pm 53.09)$ & $556.26( \pm 52.13)$ & 0.681 & \\
\hline$\Delta$ change & $-56.24( \pm 71.57)$ & & & $-24.93( \pm 59.88)$ & & & 0.737 \\
\hline BUN (mg/dL) & $11.71( \pm 0.55)$ & $11.75( \pm 0.76)$ & 0.942 & $11.84( \pm 0.58)$ & $11.83( \pm 0.56)$ & 0.991 & \\
\hline$\Delta$ change & $0.04( \pm 0.54)$ & & & $-0.01( \pm 0.66)$ & & & 0.956 \\
\hline Creatinine (mg/dL) & $0.80( \pm 0.04)$ & $0.78( \pm 0.03)$ & 0.307 & $0.76( \pm 0.02)$ & $0.73( \pm 0.02)$ & 0.015 & \\
\hline$\Delta$ change & $-0.02( \pm 0.02)$ & & & $-0.03( \pm 0.01)$ & & & 0.398 \\
\hline Albumin (g/dL) & $4.94( \pm 0.06)$ & $4.82( \pm 0.05)$ & 0.047 & $4.94( \pm 0.04)$ & $4.87( \pm 0.05)$ & 0.191 & \\
\hline$\Delta$ change & $-0.12( \pm 0.06)$ & & & $-0.07( \pm 0.06)$ & & & 0.567 \\
\hline WBC $\left(\mathrm{mm}^{3}\right)$ & $6.31( \pm 0.30)$ & $6.60( \pm 0.41)$ & 0.452 & $6.37( \pm 0.25)$ & $6.29( \pm 0.24)$ & 0.733 & \\
\hline$\Delta$ change & $0.29( \pm 0.38)$ & & & $-0.08( \pm 0.23)$ & & & 0.400 \\
\hline $\mathrm{RBC}\left(\mathrm{mm}^{3}\right)$ & $4.60( \pm 0.09)$ & $4.57( \pm 0.09)$ & 0.507 & $4.46( \pm 0.07)$ & $4.35( \pm 0.08)$ & 0.007 & \\
\hline$\Delta$ change & $-0.03( \pm 0.04)$ & & & $-0.11( \pm 0.04)$ & & & 0.166 \\
\hline HGB (g/dL) & $13.82( \pm 0.24)$ & $13.69( \pm 0.24)$ & 0.270 & $13.67( \pm 0.22)$ & $13.41( \pm 0.23)$ & 0.028 & \\
\hline$\Delta$ change & $-0.13( \pm 0.12)$ & & & $-0.27( \pm 0.11)$ & & & 0.415 \\
\hline HCT (\%) & $41.09( \pm 0.62)$ & $41.01( \pm 0.64)$ & 0.836 & $40.59( \pm 0.57)$ & $39.79( \pm 0.56)$ & 0.016 & \\
\hline$\Delta$ change & $-0.08( \pm 0.38)$ & & & $-0.80( \pm 0.31)$ & & & 0.145 \\
\hline $\operatorname{PLT}\left(10^{3} / \mu \mathrm{L}\right)$ & $258.36( \pm 11.88)$ & $247.76( \pm 13.20)$ & 0.546 & $235.67( \pm 11.64)$ & $250.81( \pm 12.08)$ & 0.014 & \\
\hline$\Delta$ change & $-10.60( \pm 9.76)$ & & & $15.15( \pm 5.77)$ & & & 0.025 \\
\hline
\end{tabular}

Data are presented as mean $\pm \mathrm{SE}$

$B U N$ blood urea nitrogen, FFA free fatty acids, HOMA-IR homeostasis model assessment, $H b$ hemoglobin, $H C T$ hematocrit, $P L T$ platelet, $R B C$ red blood cells, WBC white blood cells

*Tested using logarithmic transformation

${ }^{a} p$-values were calculated using the paired $t$-test when comparing the baseline values ( 0 weeks)

${ }^{\mathrm{b}} p$-values were calculated using the independent $t$-test when comparing the "change" values

parameters were not significantly different between the two groups.

\section{Discussion}

In our study, there was no significant difference when comparing the change values of liver disease-related parameters and serum lipid profiles in between groups.

Rhus verniciflua Stokes extract (RVSE) has been reported to have various functions including anticancer [13], antioxidant [14], and anti-inflammatory [15] properties, but its use is limited due to the presence of urushiol, an allergen in RVSE. Therefore, various methods for removing urushiol have been studied and developed $[11,16]$. In our study, urushiol-free FRVE was used according to the method used by Choi et al. [9].
The Korean Ministry of Food and Drug Safety approved daily intake of $1 \mathrm{~g}$ FRVE powder (fustin $57 \mathrm{mg} / \mathrm{g}$ ), as "it can help male climacteric health" [17]. On the other hand, there are no clinical studies on liver function. Lee et al. reported that FRVE has an anti-hepatic lipidemic effect by upregulating AMPK in cell and animal models $[8,11]$. In particular, they confirmed in an animal model that the levels of ALT, AST, TC, and TG decreased when animals with nonalcoholic fatty liver ingested FRVE [8]. ALT and AST are blood indicators that increase during liver damage and are important parameters that can predict the state of liver diseases such as hepatocellular disease or hepatitis [18]. GGT has also been reported as an indicator of internal organ fat, fatty liver $[19,20]$, and IR [21]. The liver is a major organ for 
Table 6 Dietary parameters before and 12 weeks after consuming FRVE

\begin{tabular}{|c|c|c|c|c|c|c|c|}
\hline & \multicolumn{7}{|l|}{ Group (total = 52) } \\
\hline & \multicolumn{3}{|l|}{ Control $(n=25)$} & \multicolumn{3}{|l|}{ FRVE $(n=27)$} & \multirow[b]{2}{*}{$p^{\mathrm{b}}$} \\
\hline & 0 weeks & 12 weeks & $p^{\mathrm{a}}$ & 0 weeks & 12 weeks & $p^{\mathrm{a}}$ & \\
\hline Energy intake (kcal) & $1625.72( \pm 89.40)$ & $1614.61( \pm 103.00)$ & 0.919 & $1621.91( \pm 94.29)$ & $1562.36( \pm 94.56)$ & 0.635 & \\
\hline$\Delta$ change & $-11.11( \pm 108.50)$ & & & $-59.54( \pm 124.02)$ & & & 0.772 \\
\hline Carbohydrate (g) & $225.22( \pm 13.62)$ & $205.70( \pm 11.90)$ & 0.091 & $217.18( \pm 12.14)$ & $216.01( \pm 13.94)$ & 0.941 & \\
\hline$\Delta$ change & $-19.52( \pm 11.07)$ & & & $-1.17( \pm 15.76)$ & & & 0.352 \\
\hline Protein $(\mathbf{g})$ & $64.71( \pm 6.40)$ & $70.37( \pm 5.79)$ & 0.377 & $64.15( \pm 4.97)$ & $62.65( \pm 6.24)$ & 0.855 & \\
\hline$\Delta$ change & $5.66( \pm 6.29)$ & & & $-1.50( \pm 8.15)$ & & & 0.495 \\
\hline Fat (g) & $45.39( \pm 3.78)$ & $54.34( \pm 6.06)$ & 0.198 & $52.12( \pm 5.63)$ & $48.28( \pm 4.51)$ & 0.585 & \\
\hline$\Delta$ change & $8.95( \pm 6.75)$ & & & $-3.84( \pm 6.94)$ & & & 0.194 \\
\hline Fiber (g) & $15.49( \pm 1.45)$ & $14.26( \pm 1.40)$ & 0.305 & $15.42( \pm 1.07)$ & $16.36( \pm 1.19)$ & 0.411 & \\
\hline$\Delta$ change & $-1.24( \pm 1.18)$ & & & $0.93( \pm 1.12)$ & & & 0.188 \\
\hline Vitamin A ( $\mu$ g RAE)* & $400.39( \pm 70.07)$ & $306.67( \pm 34.13)$ & 0.207 & $347.65( \pm 47.74)$ & $319.64( \pm 46.08)$ & 0.575 & \\
\hline$\Delta$ change & $-93.72( \pm 74.16)$ & & & $-28.01( \pm 75.81)$ & & & 0.539 \\
\hline Vitamin $D(\mu \mathrm{g})^{*}$ & $4.79( \pm 1.24)$ & $2.44( \pm 0.53)$ & 0.037 & $6.52( \pm 2.05)$ & $4.76( \pm 2.45)$ & 0.101 & \\
\hline$\Delta$ change & $-2.36( \pm 1.17)$ & & & $-1.76( \pm 3.19)$ & & & 0.866 \\
\hline Vitamin C (mg)* & $63.89( \pm 13.23)$ & $63.26( \pm 11.42)$ & 0.876 & $62.42( \pm 9.56)$ & $60.32( \pm 11.52)$ & 0.444 & \\
\hline$\Delta$ change & $-0.63( \pm 16.22)$ & & & $-2.10( \pm 15.07)$ & & & 0.947 \\
\hline Calcium (mg)* & $512.95( \pm 84.25)$ & $395.18( \pm 57.18)$ & 0.214 & $443.91( \pm 54.91)$ & $386.75( \pm 60.78)$ & 0.219 & \\
\hline$\Delta$ change & $-117.77( \pm 86.79)$ & & & $-57.16( \pm 85.10)$ & & & 0.620 \\
\hline Phosphate (mg) & $974.40( \pm 90.37)$ & $986.10( \pm 74.54)$ & 0.903 & $962.99( \pm 67.42)$ & $913.07( \pm 82.33)$ & 0.625 & \\
\hline$\Delta$ change & $11.70( \pm 95.07)$ & & & $-49.92( \pm 100.96)$ & & & 0.660 \\
\hline Sodium (mg) & $2648.13( \pm 211.47)$ & $3385.61( \pm 272.91)$ & 0.036 & $2937.52( \pm 247.34)$ & 3101.09 ( \pm 298.88$)$ & 0.668 & \\
\hline$\Delta$ change & $737.49( \pm 331.77)$ & & & $163.57( \pm 376.46)$ & & & 0.261 \\
\hline Cholesterol (mg) & $382.76( \pm 56.06)$ & $305.93( \pm 51.42)$ & 0.173 & $295.72( \pm 45.19)$ & $337.85( \pm 59.57)$ & 0.523 & \\
\hline$\Delta$ change & $-76.83( \pm 54.74)$ & & & $42.13( \pm 65.06)$ & & & 0.171 \\
\hline
\end{tabular}

the synthesis and metabolism of lipoproteins. If there is a dysfunction in the liver, dyslipidemia may occur, in which HDL cholesterol is lowered and triglycerides and total cholesterol are elevated [22, 23]. In particular, NAFLD is often accompanied by IR [24], obesity [25], and diabetes [26]; therefore, changes in serum lipid levels and IR are also used as auxiliary indicators to measure liver health and function. Hyperinsulinemia caused by IR induces decreased glycogen synthesis, increased fatty acid uptake in the liver, altered TG transport, inhibited fatty acid oxidation, and increased lipolysis in peripheral tissues, resulting in the accumulation of TG in the liver [27]. Therefore, the reduction of IR will help prevent and treat NAFLD. However, since there was no significant difference in the change value of IR between the test and control groups in our study, so additional confirmation through further study is warranted.

Functions of FRVE reported through other studies include anti-obesity [28], anti-cancer [29, 30], and antiinflammatory properties [5]. One animal study reported the anti-obesity effect of $R$. verniciflua leaf extract [28], but we could not confirm the effect of weight loss in humans. This is considered to be due to the large proportion of nonobese people in our study group. Previous clinical studies investigating the benefits of FRVE or RVSE only confirmed the effect on survival rate and clinical symptoms in patients with colon cancer $[29,30]$. In general, the effects of functional foods may be more effective in those who are on the border of disease or have diseases than in healthy people. Since our study was conducted on healthy adults, it may not have been 
possible to confirm the change in indicators related to liver function. In future studies, it will be necessary to verify the efficacy of FRVE in subjects with high liverrelated parameters.

This study has some limitations. We focus on healthy individuals without any diseases. Our study subjects are that the proportion of healthy and young people was high and the proportion of obese people was low. Therefore, our data cannot be generalized to subjects with any diseases. Also, the uncertain result of liver-related parameters, such as ALT, AST, and GGT levels, might be due to the small sample size and selection of the healthy subjects. In our study, the dropout rate was as high as $30 \%$ or more, which was due to reasons such as refusal to revisit due to the COVID-19 situation, personal reasons, and lack of compliance.

However, to our knowledge, this study is the first double-blind randomized controlled trial to examine liver-related parameters and lipid profile levels after FRVE intervention in humans. This study possesses several study strengths; it elucidates the efficacy of FRVE in TG and IR improvement and reports safety concerns about FRVE consumption because there were no reports of adverse effects during the 12-week intervention in 52 subjects.

In a review study, nutrients such as whole grains, polyunsaturated fatty acids, omega 3-fatty acids, vegetable proteins, and probiotics can help prevent and treat NAFLD, whereas excessive carbohydrates, simple sugars, saturated fats, trans-fatty acids, animal proteins, processed foods, and low dietary fiber intake are associated with an increase in NAFLD [4]. Therefore, as nutritional factors are very important indicators for the prevention of NAFLD, it is necessary to investigate the effects of combination with other dietary factors or at different doses of FRVE to prevent NAFLD in future studies.

\section{Conclusions}

In conclusion, no significant results could be confirmed with respect to the liver-related parameters and serum lipid profiles in our study.

\footnotetext{
Abbreviations

AMPK: AMP-activated protein kinase; ALT: Alanine aminotransferase; AST: Aspartate aminotransferase; ALP: Alkaline phosphatase; BMI: Body mass index; BUN: Blood urea nitrogen; BP: Blood pressure; CBC: Complete blood count; DBP: Diastolic blood pressure; FRV: Fermented Rhus verniciflua Stokes; FRVE: Fermented Rhus verniciflua Stokes extract; FFA: Free fatty acids; GGT: Glutamyl transferase; HDL-C: High-density lipoprotein cholesterol; Hct: Hematocrit; Hb: Hemoglobin; HOMA-IR: Homeostasis model assessment; IR: Insulin resistance; LDL-C: Low-density lipoprotein cholesterol; MDA: Malondialdehyde; NAFLD: Nonalcoholic fatty liver disease; PLT: Platelet; RVSE: Rhus verniciflua Stokes extract; RVS: Rhus verniciflua Stokes; RBC: Red blood cells; SBP: Systolic blood pressure; SPSS: Statistical Package for the Social Sciences; TC: Total cholesterol; TG: Triglycerides; WBC: White blood cells
}

\section{Supplementary Information}

The online version contains supplementary material available at https://doi. org/10.1186/s13063-021-05656-0.

Additional file 1. CONSORT 2010 Flow Diagram

Acknowledgements

We thank the subjects who participated in this study.

\section{Authors' contributions}

JHK and JKP conceptualized and designed the study; JYL acquired the data and performed the statistical analysis; JHK wrote the paper; HJL, STJ, and ML contributed to the critical revision of the manuscript; JKP accepted responsibility for the entire content of the manuscript and obtained funding for the study; and JKP supervised the study. All authors have full access to the study data and have read and agreed to the published version of the manuscript. The author(s) read and approved the final manuscript.

\section{Funding}

This research was funded by the Rural Development Administration of the Republic of Korea, grant number PJ01313503. The funding body had no role in the design of the study and collection, analysis, and interpretation of data and in writing the manuscript.

\section{Availability of data and materials}

The datasets generated and/or analyzed during the current study are not publicly available due to privacy of subjects, but are available from the corresponding author on reasonable request.

\section{Declarations}

Ethics approval and consent to participate

This study was conducted according to the guidelines of the Declaration of Helsinki and approved by the Institutional Review Board of Eulji University (IRB number: EU18-96). The study protocol was registered in the Clinical Research Information Service (CRIS: https://cris.nih.go.kr/cris/index.jsp) under number PRE20201202-005. Informed consent was obtained from all subjects who participated in the study.

\section{Consent for publication}

Not applicable.

\section{Competing interests}

The authors declare that they have no competing interests.

\section{Author details}

${ }^{1}$ Department of Food and Nutrition, Eulji University, Seongnam 13135, Gyeonggi-do, Republic of Korea. ${ }^{2}$ Department of Science in Korean Medicine, College of Korean Medicine, Graduate School, Kyung Hee University, Hoegi-dong, Dongdaemun-gu, Seoul 02435, Republic of Korea. ${ }^{3}$ Fermented Food Science Division, National Institute of Agricultural Sciences, 166, Nongsaengmyeongro, Iseo-myeon, WanjuGun, Jeollabuk-do 55365, Republic of Korea. ${ }^{4}$ Department of Food Technology and Services, Eulji University, Seongnam 13135, Gyeonggi-do, Republic of Korea.

Received: 13 April 2021 Accepted: 27 September 2021

Published online: 22 November 2021

References

1. Kang SY, Kim YJ, Park HS. Trends in the prevalence of non-alcoholic fatty liver disease and its future predictions in Korean men, 1998-2035. J Clin Med. 2020;9:1998-2035. doi: https://doi.org/10.3390/jcm9082626, PMID 32823604

2. Friedman SL, Neuschwander-Tetri BA, Rinella M, Sanyal AJ. Mechanisms of NAFLD development and therapeutic strategies. Nat Med. 2018;24:908-922. doi: https://doi.org/10.1038/s41591-018-0104-9, PMID 29967350

3. Stefan N, Häring HU, Cusi K. Non-alcoholic fatty liver disease: causes, diagnosis, cardiometabolic consequences, and treatment strategies. Lancet Diabetes Endocrinol. 2019:7:313-324. doi: https://doi.org/10.1016/S22138587(18)30154-2, PMID 30174213 
4. Perdomo CM, Frühbeck G, Escalada J. Impact of nutritional changes on nonalcoholic fatty liver disease. Nutrients. 2019;11:677. doi: https://doi.org/1 0.3390/nu11030677, PMID 30901929.

5. Choi EY, Suk KT, Choi H, Kim MK, Kwon YS, Kim MJ. Anti-inflammatory activities of fermented Rhus verniciflua stem bark extract and its growth inhibitory effect on Helicobacter pylori. Korean J Food Sci Technol. 2016; 485(5):502-7. https://doi.org/10.9721/KJFST.2016.48.5.502.

6. Kim SS, Chung JM. Taxonomic characteristics of Korean-native anacardiaceae. J Korean For Soc. 1995;84:151-65.

7. Lee SO, Kim SJ, Kim JS, Ji H, Lee EO, Lee HJ. Comparison of the main components and bioactivity of Rhus verniciflua Stokes extracts by different detoxification processing methods. BMC Complement Altern Med. 2018;18: 242. doi: https://doi.org/10.1186/s12906-018-2310-x, PMID 30165848.

8. Lee SO, Xu Y, Han H, Jeong ST, Lee YK, Paik JK, et al. Fermented Rhus verniciflua Stokes extract alleviates nonalcoholic fatty liver through the AMPK/SREBP1/PCSK9 pathway in HFD-induced nonalcoholic fatty liver animal model. Appl Sci. 2020;10(19):6833. https://doi.org/10.3390/app101 96833.

9. Choi HS, Yeo SH, Jeong ST, Choi JH, Park HS, Kim MK. Preparation and characterization of urushiol free fermented Rhus verniciflua stem bark (FRVSB) extracts. Korean J Food Sci Technol. 2012;44(2):173-8. https://doi. org/10.9721/KJFST.2012.44.2.173.

10. Nair $A B$, Jacob $S$. A simple practice guide for dose conversion between animals and human. J Basic Clin Pharm. 2016;7:27-31. doi: https://doi.org/1 0.4103/0976-0105.177703, PMID 27057123

11. Lee MS, Kim JS, Cho SM, Lee SO, Kim SH, Lee HJ. Fermented Rhus verniciflua Stokes extract exerts an antihepatic lipogenic effect in oleic-acid-induced HepG2 cells via upregulation of AMP-activated protein kinase. J Agric Food Chem. 2015;63:7270-7276. doi: https://doi.org/10.1021/acs.jafc.5b01954, PMID 26176317

12. Kim YJ, Kwon JY, Go HY, Lee DN, Ko SK, Kong KH. Study of efficacy and safety of ginseng seed oil in heathy subjects who have mild liver dysfunction: a randomized, double blinded, placebo-controlled study. J Korean Med. 2018;39:36-55. https://doi.org/10.13048/jkm.18014.

13. Lee JO, Moon JW, Lee SK, Kim SM, Kim N, Ko SG, et al. Rhus verniciflua extract modulates survival of MCF-7 breast cancer cells through the modulation of AMPK-pathway. Biol Pharm Bull. 2014;37:794-801. doi: https:// doi.org/10.1248/bpb.b13-00893, PMID 24553147.

14. Choi DR, Jeong JH, Yu KS, Lee NS, Jeong YG, Kim DK, et al. Extract of Rhus verniciflua stokes protects against renal ischemia-reperfusion injury by enhancing Nrf2-mediated induction of antioxidant enzymes. Exp Ther Med. 2018;15:3827-3835. doi: https://doi.org/10.3892/etm.2018.5913, PMID 29581741

15. Moon JE, Shin JH, Kwon O, Kim JY. A standardized extract of Rhus verniciflua Stokes protects Wistar rats against lipopolysaccharide-induced acute inflammation. J Med Food. 2015;18:1223-1230. doi: https://doi.org/10.1089/ jmf.2014.3411, PMID 26501382.

16. Liu CS, Nam TG, Han MW, Ahn SM, Choi HS, Kim TY, et al. Protective effect of detoxified Rhus verniciflua stokes on human keratinocytes and dermal fibroblasts against oxidative stress and identification of the bioactive phenolics. Biosci Biotechnol Biochem. 2013;77:1682-1688. doi: https://doi. org/10.1271/bbb.130236, PMID 23924730.

17. Ministry of Food and Drug Safety. Functional raw material information. Available from: https://www.foodsafetykorea.go.kr/portal/healthyfoodlife/sea rchHomeHFDetail.do?prdlstReportLedgNo=2017021000032548 [cited 27 Nov 2020]

18. Castera L, Friedrich-Rust M, Loomba R. Noninvasive assessment of liver disease in patients with nonalcoholic fatty liver disease. Gastroenterology. 2019;156:1264-1281.e4. doi: https://doi.org/10.1053/j.gastro.2018.12.036, PMID 30660725

19. Kim HC, Nam CM, Jee SH, Han KH, Oh DK, Suh I. Normal serum aminotransferase concentration and risk of mortality from liver diseases: prospective cohort study. BMJ. 2004;328(7446):983. doi: https://doi.org/1 0.1136/bmj.38050.593634.63, PMID 15028636.

20. Oh HJ, Kim TH, Sohn YW, Kim YS, Oh YR, Cho EY, et al. Association of serum alanine aminotransferase and $\gamma$-glutamyltransferase levels within the reference range with metabolic syndrome and nonalcoholic fatty liver disease. Korean J Hepatol. 2011;17:27-36. doi: https://doi.org/10.3350/kjhep.2 011.17.1.27, PMID 21494075.

21. Bonnet F, Ducluzeau PH, Gastaldelli A, Laville M, Anderwald CH, Konrad T, et al. Liver enzymes are associated with hepatic insulin resistance, insulin secretion, and glucagon concentration in healthy men and women Diabetes. 2011;60:1660-1667. doi: https://doi.org/10.2337/db10-1806, PMID 21521874

22. Katsiki N, Mikhailidis DP, Mantzoros CS. Non-alcoholic fatty liver disease and dyslipidemia: an update. Metabolism. 2016;65:1109-1123. doi: https://doi. org/10.1016/.j.metabol.2016.05.003, PMID 27237577.

23. Deeb A, Attia S, Mahmoud S, Elhaj G, Elfatih A. Dyslipidemia and fatty liver disease in overweight and obese children. J Obes. 2018;2018:8626818-6. https://doi.org/10.1155/2018/8626818.

24. Watt MJ, Miotto PM, De Nardo W, Montgomery MK. The liver as an endocrine organ-linking NAFLD and insulin resistance. Endocr Rev. 2019:40: 1367-1393. doi: https://doi.org/10.1210/er.2019-00034, PMID 31098621.

25. Fan JG, Kim SU, Wong WW. New trends on obesity and NAFLD in Asia. J Hepatol. 2017;67:862-873. doi: https://doi.org/10.1016/j.jhep.2017.06.003, PMID 28642059

26. Hazlehurst JM, Woods C, Marjot T, Cobbold JF, Tomlinson JW. Non-alcoholic fatty liver disease and diabetes. Metabolism. 2016;65:1096-1108. doi: https:// doi.org/10.1016/j.metabol.2016.01.001, PMID 26856933.

27. Eslamparast T, Tandon P, Raman M. Dietary composition independent of weight loss in the management of non-alcoholic fatty liver disease. Nutrients. 2017;9:800. doi: https://doi.org/10.3390/nu9080800, PMID 28933748

28. Suruga K, Tomita T, Kadokura K, Arai T. Rhus verniciflua leaf extract suppresses obesity in high-fat diet-induced obese mice. Food Nutr Res. 2019;63:29219. doi: https://doi.org/10.29219/fnr.v63.3601, PMID 31548839.

29. Lee SH, Choi WC, Yoon SW. Impact of standardized Rhus verniciflua stokes extract as complementary therapy on metastatic colorectal cancer: a Korean single-center experience. Integr Cancer Ther. 2009;8:148-152. doi: https://doi. org/10.1177/1534735409336438, PMID 19679623

30. Lee S, Kim K, Jung H, Lee S, Cheon S, Kim S, et al. Efficacy and safety of standardized allergen-removed Rhus verniciflua Stokes extract in patients with advanced or metastatic pancreatic cancer: a Korean single-center experience. Oncology. 2011;81:312-318. doi: https://doi.org/10.1159/000334 695, PMID 22179506

\section{Publisher's Note}

Springer Nature remains neutral with regard to jurisdictional claims in published maps and institutional affiliations.
Ready to submit your research? Choose BMC and benefit from:

- fast, convenient online submission

- thorough peer review by experienced researchers in your field

- rapid publication on acceptance

- support for research data, including large and complex data types

- gold Open Access which fosters wider collaboration and increased citations

- maximum visibility for your research: over $100 \mathrm{M}$ website views per year

At $\mathrm{BMC}$, research is always in progress.

Learn more biomedcentral.com/submissions 